RUNNING HEAD: PROPOSITIONAL LEARNING

\title{
A Propositional Perspective on Context Effects in Human Associative Learning
}

Jan De Houwer

Ghent University, Belgium

In press. Behavioral Processes.

mailing address:

Jan De Houwer

Ghent University

Henri Dunantlaan 2

B-9000 Ghent

Belgium

email: Jan.DeHouwer@UGent.be

phone: 003292646445

fax: 003292646489 


\begin{abstract}
Propositional models of associative learning postulate that the behavioral impact of regularities in the presence of two events is mediated by the formation of propositions about the relation between these events. Because the mere statistical contingency between events often does not provide enough information to infer the nature of the relation between those events (e.g., whether one event is a cause or an effect of the other event), it is likely that people will take into account relational information that is provided by the context when forming propositions about the relation between events. Hence, propositional models predict that contextual cues which provide relational information can moderate associative learning. The present paper provides a brief review of several studies that support this prediction. These findings contribute not only to the cognitive literature on the mental mechanisms that mediate associative learning but also to the functional literature on associative learning by providing novel evidence for arbitrarily applicable relational responding. Vice versa, functional research on relational responding can provide a new source of information for the development of cognitive theories of associative learning.
\end{abstract}

Keywords: Associative learning, propositions, context effects 


\section{A Propositional Perspective on Context Effects in Human Associative Learning}

Associative learning can be defined as a change in behavior that is due to a regularity in the presence of two events (De Houwer, Barnes-Holmes, \& Moors, 2013). The observed change in behavior, however, depends not only on the nature of the events or the regularity that binds them but also on the context in which the events occur. From a functionalcognitive perspective on learning (De Houwer, 2011; De Houwer et al., 2013), knowledge about how the context moderates associative learning not only provides important functional knowledge about the conditions under which regularities in the environment lead to changes in behavior, it also constrains cognitive theories about the mental processes that mediate the impact of regularities on behavior. The aim of these cognitive theories is twofold: (a) to account for existing functional knowledge about associative learning (i.e., their heuristic function); (b) to generate novel predictions about the conditions under which associative learning occurs (i.e., their predictive function; De Houwer et al., 2013). Hence, functional knowledge about (context and other effects in) associative learning provides the knowledge base with which cognitive theories can be evaluated.

During the past forty years or so, most cognitive theories of associative learning centered on the idea of association formation. According to association formation theories, regularities in the presence of two events can result in the formation of an association between the mental representations of those events. Once this association has been formed, the presence of one event not only activates the representation of this event but also the representation of the other event, which in its turn can lead to a change in behavior (e.g., Dickinson, 2012). Hence, associations can be conceived of as hypothetical mental structures akin to wires or tubes via which activation can spread between mental representations (Mitchell, De Houwer, \& Lovibond, 2009). 
Recently, my colleagues and I proposed that associative learning in humans is mediated by the formation of propositions rather than associations (De Houwer, 2009; Mitchell et al., 2009). Propositions can be conceived of as informational units in memory that encode statements about the world. Importantly, propositions about relations between events in the world can refer not only to the events themselves but also to how those events are related. Hence, there are two fundamental differences between associations and propositions: (a) only propositions can be true or false (because only propositions are statements whereas associations are structures) and (b) only propositions specify information about how events are related (see Lagnado, Waldmann, Hagmayer, \& Sloman, 2007, for an excellent discussion of this issue).

In the present paper, I focus on the second unique aspect of propositions and discuss its implications for research on context effects in associative learning. In a first section, I briefly explain why, from the perspective of propositional models, context can be a crucial moderator of associative learning. In the second section, I review a number of recent studies that reveal an impact of contextual relational information on associative learning. Whereas the arguments put forward in the first two sections originate from the cognitive literature on associative learning and are formulated in terms that are part of that literature (e.g., information, formation of propositions), in the third section I attempt to link cognitive research on the role of contextual relational information to phenomena that have been documented in the functional literature. More specifically, I argue that the cognitive studies that are summarized in Section 2 provide new evidence for the functional phenomenon of (arbitrarily applicable) relational responding. This novel perspective highlights a common ground that allows for mutually supportive interactions between functional and cognitive learning researchers. 


\section{Context is Vital for the Formation of Propositions}

Regularities in the presence of two events most often do not contain enough information to determine the way in which those events are related. Consider the following example (Lagnado et al., 2007; Waldmann \& Holyoak, 1992): A patient is treated in hospital for a mysterious disease X. The doctor in charge asks for a blood test which shows that the patient has a particular chemical substance $\mathrm{Y}$ in his blood. Based on the mere co-occurrence of disease $\mathrm{X}$ and substance $\mathrm{Y}$, it is not possible to determine how the two are related. For instance, substance Y might be the cause of disease X or it might be an effect of disease X. Knowing how disease $\mathrm{X}$ and substance $\mathrm{Y}$ are related is, however, vital for determining the action that the doctor should take: If substance $\mathrm{Y}$ is the cause of disease $\mathrm{X}$, then the patient might be cured by removing substance $\mathrm{Y}$ from the blood. If substance $\mathrm{Y}$ is an effect of disease X, such a course of action would have little effect on the disease.

Because (a) it can be important to determine how events are related and (b) the statistical regularity between events often does not provide enough information to determine the nature of the relation between events, it makes sense to assume that people will use whatever information is available in the context that can help them determine how events in the world are related. Contextual relational information might be provided not only by contextual cues that are present simultaneously with the paired events (e.g., another stimulus that might cause the presence of substance $\mathrm{Y}$ ) but also by events and regularities in the past (e.g., prior experiences with other chemical substances as causes or effects of diseases). If the context (and more specifically, elements of the context that provide relational information) plays a vital role in the formation of propositions, and if human associative learning is mediated by the formation of propositions about the relation between events, then it follows that human associative learning can be moderated by aspects of the context that 
provide information about the nature of the relation between events. Evidence in line with this prediction has been obtained in a number of studies that are summarized in the next section.

\section{Empirical Evidence}

In the cognitive literature on the effects of contextual relational information, one can distinguish two lines of research based on the elements in the context that carry the relational information. In a first set of studies, relational information was implied by regularities between filler stimuli that were intermixed with target stimuli. In the second set of studies, relational information was provided by verbal instructions. The review is not meant to be exhaustive (e.g., see, for instance, De Houwer, Beckers, \& Glautier, 2002, Waldmann, 2000, and Waldmann \& Holyoak, 1992, for other relevant studies) but merely aims to illustrate some of the relevant findings.

\section{Other Regularities as a Context}

Shanks and Darby (1998, Experiment 2) showed participants a series of (fictitious) allergy tests that had been administrated to a group of patients with identical food-allergies. Each test record specified the type(s) of food that a patient had eaten. After inspecting a test record, participants predicted whether the patient would show an allergic reaction. On training trials, feedback was given about whether the prediction was correct. During these training trials, different filler cues (i.e., types of food) were presented both in isolation and in compound with another cue. For some filler cues, the relation with the outcome (i.e., allergic reaction present or absent) followed a positive patterning design (i.e., $\mathrm{A}+, \mathrm{B}+, \mathrm{AB}-, \mathrm{E}+, \mathrm{F}+$, EF-) whereas for others filler cues, it followed a negative patterning design (i.e., C-, D-, CD+, G-, H-, GH+; where different letters stand for different foods, “+” stands for the presence of an allergic reaction, and “_“ stands for the absence of an allergic reaction). These 
filler trials were meant to provide a context that carries the following relational information: "the allergic effect of a food is reversed when the food is presented alone compared to when it is presented together with another food”. Importantly, during training, target cues were also presented. Some target cues always occurred alone (i.e., I+, J+, M-, N-) whereas other target cues were always presented in compound (i.e., KL-, OP+).

On subsequent test trials, participants continued to make predictions but feedback was no longer provided. The crucial test trials were those in which the target cues were presented in ways not seen before (i.e., IJ, MN, K, L, O, and P). Participants who learned to make the correct predictions on the filler trials, also responded to the crucial test trials in line with the relational information that was implied by the filler trials. More specifically, these participants predicted the outcome less often for target cues that were previously paired with the outcome (i.e., on IJ, O, and P test trials that followed $\mathrm{I}+$, $\mathrm{J}+$, and $\mathrm{OP}+$ training trials) than for target cues that were never paired with the outcome during training (i.e., $\mathrm{MN}, \mathrm{K}$, and $\mathrm{L}$ test trials that followed M-, N-, and KL- training trials). According to propositional models of associative learning, participants used the relational information implied by the filler trials to form propositions about the relation between the test cues and the outcome. For instance, based on I+ and J+ trials, they formed the proposition that I and J together would not be followed by the outcome and therefore predicted that the compound IJ would not be followed by the outcome.

My colleagues and I replicated and extended these findings in a number of studies that were reported in two papers (De Houwer \& Vandorpe, 2010; Zanon, Gast, \& De Houwer, 2010; see Wills, Graham, Koh, McLaren, \& Rolland, 2011, for related studies). In a first study (De Houwer \& Vandorpe, 2010), we found an impact of the relational information that was implied by the filler trials even when knowledge about the target-cue outcome relations 
was assessed using an Implicit Association Test (IAT). The IAT is a reaction time task in which participants classify stimuli into one of four categories by pressing one of two keys. The basic idea behind the IAT is that classification performance should be superior when the categories assigned to the same key are somehow related in memory than when they are unrelated (see Greenwald, McGhee, \& Schwartz, 1998). Because the IAT requires the use of four stimulus categories, we had to alter the design of Shanks and Darby (1998) slightly. Rather than having one outcome that could be present or absent, we paired cues with one of two possible outcomes. More specifically, cues were said to be chemical substances that could either result in nausea or skin irritation. In a first between-subjects condition (Condition Same), filler cues were followed by the same outcome independent of whether they were presented in isolation or in compound (i.e., A-O1, B-O1, AB-O1, C-O2, D-O2, CD-O2, where different letters refer to different chemical substances, O1 refers to the first outcome, and $\mathrm{O} 2$ refers to the second outcome). In a second condition (Condition Opposite), cues were followed by a different outcome when presented in isolation than when presented in compound (i.e., A-O1, B-O1, AB-O2, C-O2, D-O2, CD-O1). During training, participants in both conditions saw the target compound $\mathrm{EH}$ together with $\mathrm{O} 1$ and the target compound GH with $\mathrm{O} 2$.

In Condition Same, the contextual relational information that was embedded in the filler trials allowed participants to infer that $\mathrm{E}$ would be followed by $\mathrm{O} 1$ when presented in isolation whereas $\mathrm{G}$ on its own would be followed by O2. Performance on the IAT confirmed that participants found it easier to respond when E and O1 were assigned to one key and G and $\mathrm{O} 2$ were assigned to the second key than when the key assignments were reversed (i.e., press one key for E and $\mathrm{O} 2$ and second key for G and O1). In Condition Opposite, however, the contextual relational information implied that $\mathrm{E}$ on its own would be followed by $\mathrm{O} 2$ and 
$\mathrm{G}$ on its own by $\mathrm{O} 1$ even though participants actually experienced trials on which $\mathrm{E}$ was paired with $\mathrm{O} 1$ and $\mathrm{G}$ was paired with $\mathrm{O} 2$. In line with the prediction of propositional models, IAT performance showed that participants found it easier to relate E with O2 and G with O1 than to relate E with $\mathrm{O} 1$ and $\mathrm{G}$ with $\mathrm{O} 2$. In sum, in Condition Opposite, associative learning did not reflect the actual target-cue pairings but the relational information that was embedded in the filler trials.

In a recent set of studies, my colleagues and I showed that associative learning of evaluations can also be moderated by contextual relational information (Zanon, De Houwer, \& Ga st, 2012). We implemented the same design as De Houwer and Vandorpe (2010) but used a positive and negative event as outcomes. More specifically, O1 corresponded to winning points whereas $\mathrm{O} 2$ corresponded to the loss of points. Hence, participants experienced events in which cue E co-occurred with winning (i.e., EF-win trials) and cue G co-occurred with losing (GH-loss trials). Across three different studies, we used three different reaction time measures that each provided an indirect index of how much participants liked the target cues $\mathrm{E}$ and $\mathrm{G}$. In each study, we found that $\mathrm{E}$ was liked more than G in Condition Same. In Condition Opposite, however, no difference was found in the liking of $\mathrm{E}$ and $\mathrm{G}$. Hence, the relational information provided by the filler trials moderated the impact of the stimulus pairings on the liking of the target cues. Nevertheless, the fact that the liking of E and G did not reverse in Condition Opposite provides a first indication that there are boundary conditions to the impact of contextual relational information on associative learning. The studies that are discussed in the next section shed some light on what those boundary conditions might be.

\section{Verbal Instructions as a Context}


In the studies of Peters and Gawronski (2011), participants were asked to form an impression of four previously unknown persons based on information about the traits of these persons. Pictures of Persons A and B were most often presented together with positive trait words whereas pictures of Persons C and D were most often paired with negative trait words. Participants were told that the trait words gave a true description of Persons A and C (e.g., if Person A was paired with the word FRIENDLY, it implied that Person A was friendly) whereas Persons B and D possessed the traits that were opposite to those implied by the trait word (e.g., if Person B was presented with the word FRIENDLY, it implied that Person B was unfriendly). After this training phase, evaluations of the four target persons were assessed using both evaluative ratings and two indirect measurement tasks (i.e., the evaluative priming task and the affect misattribution task) that were designed to assess automatic evaluations (i.e., evaluative responses that were evoked by the target persons very quickly and even though this was not required by the task at hand; see De Houwer, Gawronski, \& Barnes-Holmes, in press). If changes in liking are merely a function of actual stimulus pairings, one would expect that participants like Persons A and B more than Persons $\mathrm{C}$ and $\mathrm{D}$, regardless of the relational information that was presented. Whereas Person A was indeed liked more than Person C, results showed that Person B was liked less than Person D, even though B was paired with positive stimuli whereas C co-occurred with negative stimuli. One might argue that the impact of contextual relational information on evaluative ratings is not particularly surprising given that participants had ample time to take into account the relational information provided by the instructions. It is important to note, however, that also automatic evaluations as captured by indirect measurement tasks were more negative for Person B than for Person D. 
In their third study, Peters and Gawronski (2011) discovered that the impact of verbal relational information depends on the time at which this information presented. If participants were told before the training phase which person-trait pairings were true and which should be reversed, then both the evaluative ratings and the automatic evaluations were in line with the relational information (i.e., A liked more than C but B liked less than D). If, however, participants first saw all person-trait pairings and were told only afterwards which pairings presented true information and which should be reversed, then automatic evaluations were influenced less by the relational information than evaluative ratings. More specifically, whereas participants rated $\mathrm{A}$ as more positive than $\mathrm{C}$ and $\mathrm{B}$ as less positive than $\mathrm{D}$, automatic evaluations revealed a preference for A over C but no difference in the evaluation of B and D. This finding also sheds some light on the results of Zanon et al. (2012) that were described in the previous section. They also found that contextual relational information eliminated but did not reverse the effect of stimulus pairings on automatic evaluations. The lack of a reversal might have been due to the fact that the relational information was carried by the filler trials which were scattered throughout the learning phase. Hence, the relational information probably became available only after at least some target-outcome pairings were presented.

In a conceptual replication of the third study of Peters and Gawrsonki (2011), my colleagues and I repeatedly paired one neutral nonword (e.g., LOKANTA - HAPPY) with an existing positive word and a second neutral nonword with an existing negative word (e.g., FEVKANI - UGLY; Zanon, De Houwer, Gast, \& Smith, in press, Experiment 1). Either before or after the pairings, participants were told that the nonwords were antonyms of the existing words, that is, that each nonword had the opposite meaning of the existing word with which it was paired. At the end of the experiment, automatic evaluations of the nonwords were captured using a version of the IAT. When the antonym instructions were presented 
before the nonword-word pairings, the impact of the nonword-word pairings was reversed (i.e., participants liked the nonword that was paired with the positive word less than the nonword that was paired with the negative word). In line with the findings of Peters and Gawronski, the effect of the stimulus pairings was merely attenuated but not reversed when the antonym instructions was presented after the pairings.

In a second study (Zanon et al., in press, Experiment 2), we tested one possible explanation for why the impact of relational information depends on the time at which that information is presented. Given that in daily life, stimuli that are somehow similar tend to cooccur more often than stimuli that are dissimilar, it might be the case that - in the absence of any other contextual relational information - the mere pairing of two stimuli is seen as a cue that those stimuli are somehow equivalent (see Hayes, Barnes-Holmes, \& Roche, 2001, for a discussion of this assumption). For instance, when participants see a picture of an unknown person together with a trait word (as was the case in the studies of Peters and Gawronski, 2011), they might infer that the trait word provides a true reflection of the properties of the depicted person. However, they will not draw these inferences if other relational information is available that contradicts the default assumption (e.g., the verbal message the persons possess the traits opposite to those implied by the trait words). When they receive this relational information only after the pairings, they can form new propositions about the target persons based on this new relational information but they cannot erase the propositions that they formed on the basis of the default assumption earlier on during the training phase. If one adds the final assumption that these initial propositions have a larger effect on automatic evaluations than on evaluative ratings (e.g., because the former are more difficult to control), one can explain the fact that relational information has less impact on automatic evaluations than on evaluative ratings if that information is presented only after the pairings. 
To test this idea, we presented verbal relational information both before and after the stimulus pairings. Participants could be told that the nonwords and words were either synonyms or antonyms, thus resulting in four conditions (i.e., synonym before and after, antonyms before and after, synonyms before and antonyms after, antonyms before and synonyms after). We also ran a condition in which verbal relational information was not provided, neither before nor after the pairings. As in earlier studies, we registered both evaluative ratings and automatic evaluations. In line with the account outlined above, automatic evaluations were influenced more strongly by the initial relational information than evaluative ratings. Moreover, results in the condition without any relational information were similar to those in the synonym-before-and-after condition, thus suggesting that in the absence of contradictory contextual relational information, mere stimulus pairings are seen as a cue for the equivalence of the paired stimuli.

\section{A Functional-Cognitive Analysis of the Empirical Evidence}

The studies described above are firmly rooted in the cognitive tradition of learning research. Within this cognitive approach, the main aim is to uncover the mental mechanisms that mediate (associative) learning, that is, the mechanisms by which regularities in the environment produce changes in behavior. For instance, the studies listed above were designed primarily to evaluate propositional models of associative learning. However, from a functional-cognitive perspective (De Houwer, 2011), cognitive and functional research although driven by fundamentally different objectives - can be mutually supportive. On the one hand, cognitive research produces new functional knowledge (i.e., new knowledge about the conditions under which behavioral effects occur). On the other hand, functional research constrains cognitive theories because it generates functional knowledge that cognitive theories should be able to explain. In other words, cognitive theories can inspire the discovery 
of novel functional knowledge whereas functional research can provide additional input for the development of cognitive theories.

The cognitive and functional approach can, however, interact only to the extent that there is a common language to describe the research that each approach generates. Elsewhere, my colleagues and I proposed that this common language can be found in the functional approach. Hence, we have argued that cognitive researchers can benefit from describing in functional terms the behavioral effects that they examine and the data they generate (De Houwer, 2011; De Houwer et al., 2013; De Houwer et al., in press). In this section, I will attempt to provide such a functional analysis of the cognitive studies that were described in the previous section. In doing so, I hope to clarify not only how this cognitive research contributes to the functional approach, but also how existing functional research can further constrain cognitive theories of associative learning.

In functional terms, studies on the impact of contextual relational information provide additional evidence for a phenomenon known as context dependent relational responding. Consider the study by Steele and Hayes (1991). During a first training phase, participants were shown a sample stimulus (e.g., a short line) together with two comparison stimuli (e.g., a short line and a long line), one of which was physically identical to the sample. In the presence of a first arbitrary relational cue (a meaningless symbol; i.e., cue SAME), participants were reinforced for selecting the comparison stimulus that was identical to the sample (e.g., the short line) whereas in the presence of a second relational cue (another meaningless symbol; i.e., cue OPPOSITE), the selection of the other stimulus was reinforced (e.g., the long line). The fact that participants were able to respond in the desired way could be seen as an example of context dependent non-arbitrary relational responding. Participants responded relationally in that their choices were based not on the properties of one stimulus 
as such (i.e., the identity of the short line) but on the relation between the identity of the sample and the identity of the comparison stimuli. Relational responding could have been non-arbitrary in that is was based on the physical properties of the stimuli. Most important for the present purposes, relational responding was context dependent in that a contextual cue (SAME or OPPOSITE) determined the type of relation that controlled the choices.

The study of Steele and Hayes (1991) did not end there. During a second training phase, sample and comparison stimuli were arbitrarily selected such that they did not (systematically) share any physical resemblance. The stimuli were randomly assigned to different arrays and were denoted by a letter-digit code in which the letter referred to the array to which the stimulus belonged and the digit referred to the location of the stimulus within the array (e.g., stimulus B2 refers to the second stimulus in array B). Importantly, when cue OPPOSITE was present, participants were reinforced for selecting B3 from an array of comparison stimuli in the presence of A1 and for selecting C3 from another array of comparison stimuli in the presence of A1. When relational cue SAME was present, however, B1 and C1 had to be selected in the presence of A1. During the crucial test trials, participants saw B3 as a sample stimulus and C1 and C3 as comparison stimuli. In the presence of the relational cue SAME, participants selected C3 whereas in the presence of relational cue OPPOSITE, participants selected C1 even though participants had never been reinforced for making these choices. These results demonstrate that participants responded relationally (i.e., because the choices were not based merely on one stimulus but on the relation between the sample and comparison stimuli) in a way that was context dependent (because relational responding was dependent on the context cue) and based on arbitrary stimulus properties (because the stimuli did not share any systematic physical relation). This behavioral 
phenomenon is commonly referred to as arbitrarily applicable relational responding (see Dymond \& Roche, 2013, and Hayes et al., 2001, for relevant reviews). ${ }^{1}$

The cognitive studies on the impact of contextual relational information that were described in the second section of this paper share important features with study of Steele and Hayes. Most importantly, responding in the cognitive studies was most likely relational in nature. Consider the study of Peters and Gawronski (2011) in which participants liked a person whose picture was paired with positive trait words unless the traits were said to describe the opposite of the person's actual traits. In all likelihood, (evaluative) responding to the target person was not based merely on the properties of the target person, including the properties of the person that might have been changed as a result of the picture-trait pairings. If responding was non-relational, then why would relational cues (e.g., verbal instructions about the relation a person and the trait words it was paired with) have such a big impact on responding to the person? Likewise, in the studies of Shanks and Darby (1998; also see De Houwer \& Vandorpe, 2010) the filler trials provide a relational context that specifies the functional relation between the consequences that follow single versus compound stimulus presentations (i.e., same outcome or opposite outcomes). When participants subsequently encountered novel stimuli during the test trials, responding to the test stimuli is relational in that it is controlled by this relational context.

Of course, any functional analysis of any individual behavioral finding can be submitted to debate and further testing. With regard to our functional analysis of the results of

\footnotetext{
1 Hayes et al. (2001) argued that once a person has developed the ability to respond relationally in an arbitrarily applicable manner, all types of (conditioned) responding would qualify as arbitrarily applicable relational responding, even those instances in which people seem to respond to nonarbitrary properties of stimuli or stimulus relations. Hence, all types of (conditioned) responding should be sensitive to control by contextual relational cues.
} 
Peters and Gawrsonki (2011), for instance, one could argue that the properties of the stimulus persons did somehow change as the result of the direct contingencies between the pictures and trait words and did so in a way that was modulated by the contextual relational cues. Assume, for instance, that a stimulus person acquires the valence that is implied by the contextual meaning of the trait word with which it is paired (e.g., the trait word “ happy” in the context of the relational cue "false" might be recoded as "sad", thus leading to a disliking of persons paired with the trait word "happy”). Although this account implies context dependent relational responding to the trait word, the change in responding to the stimulus person might still be considered as non-relational (see Zanon et al., in press, for a discussion). One way to argue against such a non-relational account of the results of Peters and Gawronski would be to test whether the changes in evaluative responding to a person transfer to stimuli that were not paired with the trait words but that do participate in relation with that person (e.g., that the same changes occur for friends of that person but opposite changes occur for enemies of that person; see Smyth, Barnes-Holmes, \& Forsyth, 2006, ValdiviaSalas, Dougher, \& Luciano, 2013, and Whelan \& Barnes-Holmes, 2004, for more details about this approach). Regardless of the outcomes of this additional research, it seems at least reasonable to raise the possibility that cognitive studies on the impact of contextual relational information provide new evidence for the functional phenomenon of (arbitrarily applicable) relational responding. Moreover, cognitive studies could inspire functional researchers to engage in quasi-replications of these studies using training and testing methods that provide more control over the learning history that participants bring to bear during the experiment. In sum, cognitive research could contribute to the functional literature on associative learning in different ways. 
By linking the cognitive studies on contextual relational information to the functional phenomenon of relational responding, it also becomes apparent that existing functional research on relational responding could be relevant for the development of cognitive theories of associative learning. In order to explain the fact that humans can respond on the basis of relations between stimuli and can do so in a context dependent manner, it seems necessary to assume that the cognitive mechanisms that mediate these behavioral effects somehow process information about the way in which stimuli are related. In other words, evidence for context dependent relational responding supports the hypothesis that propositions mediate associative learning. In any case, current or future cognitive theories of associative learning should be able to account for context dependent (non-arbitrary or arbitrarily applicable) relational responding. Because of the unfortunate divide between functional and cognitive approaches in psychology, this challenge has rarely if ever been addressed by cognitive models of associative learning. In sum, cognitive research on associative learning can benefit from venturing into the functional domain just like functional research can profit from cognitive research.

\section{Conclusion}

As is evidenced by the papers reported in this special issue, the effects of context on associative learning have been studied extensively. The present paper highlights a number of studies on the impact of contextual relational information that were inspired by propositional models of human associative learning. From the perspective of propositional models, contextual relational information should play a vital role in associative learning because it shapes the propositions that are thought to mediate learning. Various studies confirmed that contextual relational information moderates associative learning, irrespective of whether relational information was embedded in other regularities or in verbal instructions. In some 
cases, effects of stimulus pairings were even reversed as the result of the presence of contextual relational cues. These findings do not only support propositional models of associative learning but also contribute to the functional literature by providing new evidence for arbitrarily applicable relational responding. Vice versa, existing functional research on relational responding can inform cognitive research about the mental mechanisms that mediate associative learning. Research on the impact of contextual relational cues is thus likely to provide fertile common ground for both cognitive and functional scholars of associative learning. 


\section{References}

Dickinson, A. (2012). Associative learning and animal cognition. Philosophical Transactions of The Royal Society B, 367, 2733-2742.

De Houwer, J. (2009). The propositional approach to associative learning as an alternative for association formation models. Learning \& Behavior, 37, 1-20.

De Houwer, J. (2011). Why the cognitive approach in psychology would profit from a functional approach and vice versa. Perspectives on Psychological Science, 6, 202-209.

De Houwer, J., Barnes-Holmes, D., \& Moors, A. (2013). What is learning? On the nature and merits of a functional definition of learning. Psychonomic Bulletin \& Review, 20, 631-642.

De Houwer, J., Beckers, T., \& Glautier, S. (2002). Outcome and cue properties modulate blocking. Quarterly Journal of Experimental Psychology, 55A, 965-985.

De Houwer, J., Gawronski, B., \& Barnes-Holmes, D. (in press). A functionalcognitive framework for attitude research. European Review of Social Psychology.

De Houwer, J., \& Vandorpe, S. (2010). Using the Implicit Association Test as a measure of causal learning does not eliminate effects of rule learning. Experimental Psychology, 57, 61-67.

Dymond, S., \& Roche, B. (Eds.) (2013). Advances in Relational Frame Theory: Research and Application. Oakland, CA: New Harbinger.

Greenwald, A. G., McGhee, D. E., \& Schwartz, J. L. K. (1998). Measuring individual differences in implicit cognition: The Implicit Association Test. Journal of Personality and Social Psychology, 74, 1464-1480.

Hayes, S. C., Barnes-Holmes, D., \& Roche, B. (Eds.). (2001). Relational Frame Theory: A Post-Skinnerian account of human language and cognition. New York: Plenum 
Press.

Lagnado, D. A., Waldmann, M. R., Hagmayer, Y., \& Sloman, S. A. (2007). Beyond covariation: Cues to causal structure. In A. Gopnik \& L. Schulz (Eds.), Causal learning: Psychology, philosophy, and computation (pp. 154-172). Oxford: Oxford University Press.

Mitchell, C. J., De Houwer, J., \& Lovibond, P. F. (2009). The propositional nature of human associative learning. Behavioral and Brain Sciences, 32, 183-198.

Peters, K. R., \& Gawronski, B. (2011). Are we puppets on a string? Comparing the impact of contingency and validity on implicit and explicit evaluations. Personality and Social Psychology Bulletin, 37, 557-569.

Shanks, D. R., \& Darby, R. J. (1998). Feature- and rule-based generalization in human associative learning. Journal of Experimental Psychology: Animal Behavior Processes, 24, 405-415.

Smyth, S., Barnes-Holmes, D., \& Forsyth, J. P. (2006). A derived transfer of simple discrimination and self-reported arousal functions in spider fearful and non-spider fearful participants. Journal of the Experimental Analysis of Behavior, 85, 223-246.

Steele D. L, \& Hayes S. C. (1991). Stimulus equivalence and arbitrarily applicable relational responding. Journal of the Experimental Analysis of Behavior, 56, 519-555.

Valdivia-Salas, S., Dougher, M., \& Luciano, C. (2013). Derived relations and generalized alteration of preferences. Learning and Behavior, 41, 205-217.

Waldmann, M. R. (2000). Competition among causes but not effects in predictive and diagnostic learning. Journal of Experimental Psychology: Learning, Memory, and Cognition, 26, 53-76.

Waldmann, M. R., \& Holyoak, K. J. (1992). Predictive and diagnostic learning within causal models: Asymmetries in cue competition. Journal of Experimental Psychology: 
General, 121, 222-236.

Whelan, R., \& Barnes-Holmes, D. (2004). The transformation of consequential functions in accordance with the relational frames of same and opposite. Journal of the Experimental Analysis of Behavior, 82, 177-195.

Wills, A.J., Graham, S., Koh, Z., McLaren, I.P.L. and Rolland, M.D. (2011). Effects of Concurrent Load on Feature- and Rule-based Generalization in Human Contingency Learning. Journal of Experimental Psychology: Animal Behavior Processes, 37, 308-316.

Zanon, R., De Houwer, J., \& Gast, A. (2012). Context effects in evaluative conditioning of implicit evaluations. Learning \& Motivation, 43, 155-165.

Zanon, R., De Houwer, J., Gast, A., \& Smith, C. A. (in press). When does relational information influence evaluative conditioning. Quarterly Journal of Experimental Psychology. 
Propositional Learning 23

\section{Author Note}

Jan De Houwer, Ghent University, Belgium. The preparation of this paper was made possible by Methusalem Grant BOF09/01M00209 of Ghent University to Jan De Houwer. I thank Sean Hughes for his comments on an earlier draft of this paper and his help in addressing the comments of the reviewers. Correspondence concerning this paper can be addressed to Jan De Houwer, Ghent University, Henri Dunantlaan 2, B-9000 Ghent, Belgium. Electronic mail can be sent to Jan.DeHouwer@UGent.be . 